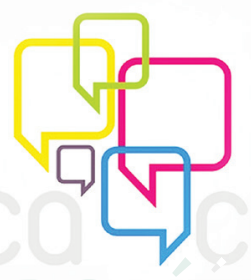

\title{
Fenomenologias da cibercultura e da convergência digital nos mass media: a constituição da Webrádio FIP ${ }^{1}$
}

Phenomenology of cyberculture and digital convergence in the media: the constitution of FIP Webradio

Fenomenología de la cibercultura y la convergencia digital en los medios de comunicación: la constitución de la FIP Webradio

Josiane Santos Brant ROCHA ${ }^{2}$ Vivianne Margareth Chaves PEREIRA REIS ${ }^{3}$ Gustavo Souza SANTOS ${ }^{4}$ Alexandre Rodrigues COSTA FILHO ${ }^{5}$ Bruna Carvalho Ferreira dos SANTOS ${ }^{6}$ Lucas Lourenço Sampaio LOPES ${ }^{7}$ Maxmiller Francisco dos SANTOS ${ }^{8}$ Thaís Figueredo LOPES ${ }^{9}$

\section{RESUMO}

O universo da cibercultura e a evolutiva tecnológica têm instaurado um novo tempo na sociedade da comunicação. O presente trabalho parte desta análise na medida em que estuda a convergência digital. O objetivo foi desenvolver um projeto experimental de webrádio para a Rádio FIP, veículo acadêmico das Faculdades Integradas Pitágoras de Montes Claros - FIPMoc. Os métodos incluíram uma pesquisa documental tendo por extrato webrádios acadêmicas nacionais. A partir do estudo documental e da fecundidade literária, construiu-se um projeto de programação e constituição para o veículo. Percebese a possibilidade de criação de espaços privilegiados de interação e comunicação social nutrindo-se

1 Trabalho apresentado à sexta edição da Revista Ação Midiática - Estudos em Comunicação, Sociedade e Cultura, publicação ligada ao Programa de Pós-Graduação em Comunicação, da Universidade Federal do Paraná.

2 Doutora em Ciência do Desporto pela Universidade Trás-os-Montes e Alto Douro. Docente na Universidade Estadual de Montes Claros - Unimontes e Faculdades Integradas Pitágoras de Montes Claros - FIPMoc. Membro da Equipe de Pesquisa do CEAD/Unimontes. josianenat@yahoo.com.br

3 Mestre em avaliação fisica nas ativ. fisicas e desportivas pela Universidade de Trás-os-Montes e Alto Douro (2008) revalidação da Universidade Federal do Rio Grande Norte (UFRN) como Mestre em Ciência da Saúde e Pós graduada em ginástica de academia. Membro da Equipe de Pesquisa do Centro de Educação a Distância da Universidade Estadual de Montes Claros - CEAD/Unimontes e docente nas Faculdades Unidas do Norte de Minas - FUNORTE. viola.chaves@yahoo.com.br

4 Graduando em Comunicação Social - Publicidade e Propaganda pelas Faculdades Integradas Pitágoras de Montes Claros FIPMoc e membro da Equipe de Pesquisa do Centro de Educação a Distância da Universidade Estadual de Montes Claros - CEAD/Unimontes. gustavo.ccpv@gmail.com

5 Graduando em Comunicação Social - Publicidade e Propaganda pelas Faculdades Integradas Pitágoras de Montes Claros FIPMoc. alexandre@boleteen.com

6 Graduanda em Comunicação Social - Publicidade e Propaganda pelas Faculdades Integradas Pitágoras de Montes Claros - FIPMoc. brunacfscarvalho@gmail.com

7 Graduando em Comunicação Social - Publicidade e Propaganda pelas Faculdades Integradas Pitágoras de Montes Claros - FIPMoc. lucaslslopes@gmail.com

8 Graduando em Comunicação Social - Publicidade e Propaganda pelas Faculdades Integradas Pitágoras de Montes Claros - FIPMoc. maxmiller.santos@gmail.com

9 Graduanda em Comunicação Social - Publicidade e Propaganda pelas Faculdades Integradas Pitágoras de Montes Claros - FIPMoc. thaislopestm@hotmail.com 


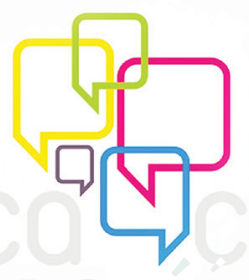

ação midiáática

N. 6 | Ano 2013

Universidade Federal do Paraná | Programa de Pós-Graduação em Comunicação

dos sulcos da cibercultura e da história dos mass media, como o rádio.

Palavras-chave: Webrádio; Rádio universitária; Cibercultura; Convergência digital; Radiodifusão.

\begin{abstract}
The universe of cyberspace and technological evolution have brought a new era in communication society. This part of the analysis work in that studies the digital convergence. The goal was to develop an experimental project webradio for FIP Radio, academic Vehicle Integrated Colleges Pythagoras Montes Claros - FIPMoc. The methods included desk research by taking extract national academic webradios. From the desk study and literary fecundity, we constructed a programming project and constitution for the vehicle. We can see the possibility of creating privileged spaces for social interaction and communication nourishing the grooves of cyberculture and the history of mass media such as radio.
\end{abstract}

Keywords: Webradio; College radio; Cyberculture; Digital convergence; Broadcasting.

\title{
RESUMEN
}

El universo del ciberespacio y la evolución tecnológica han traído una nueva era en la sociedad de la comunicación. Esta parte del trabajo de análisis en el que estudia la convergencia digital. El objetivo era desarrollar un proyecto experimental para la webradio FIP Radio, Vehículo académica integrada Colegios Pitágoras Montes Claros - FIPMoc. Los métodos incluyen la investigación documental tomando extracto webradios académicas nacionales. Desde el estudio teórico y la fecundidad literaria, hemos construido un proyecto de programación y la constitución para el vehículo. Podemos ver la posibilidad de crear espacios privilegiados para la interacción social y la comunicación nutrir las ranuras de la cibercultura y la historia de los medios de comunicación como la radio.

Palabras clave: Webradio; La radio de la universidad; Cibercultura; La convergencia digital; Radiodifusión.

\section{Introdução}

Os media tem assumido um papel incisivo na sociedade, influenciando relações, atitudes e comportamentos humanos (SAMPAIO, 2008). Na internet, os meios de comunicação tornaram-se oásis de interação humana e palco de novas práticas de sociabilidade (TEIXEIRA; SILVA, 2009). Nesse contexto, um dos destaques da pirotecnia tecnológica foi o rádio, que de maneira admirável modelou seus eixos comunicacionais no universo dos sujeitos no tempo e no espaço (VIGIL, 2003). Desde os primórdios de sua constituição, o meio radiofônico inaugurou uma modalidade de comunicação acalorada, intensa e intempestivamente social (KISCHINVSKY, 2007), posicionando-se para além de um meio de comunicação, mas como uma companhia conhecida e próxima (VIGIL, 2003).

Há cenários robustos que apontam para grandes modificações sociais. A convergência digital e os amplos prospectos nos quais os meios de comunicação e os indivíduos se inserem atingem cada fragmento da sociedade contemporânea. O rádio é parte desse novo dado social e tecnológico (TEIXEIRA; SILVA, 2009). Nesse sentido, o rádio, como os demais meios 


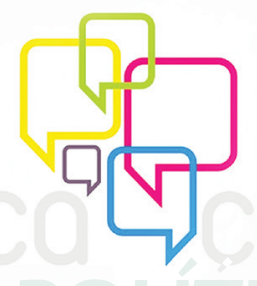

açãô I0 mididiática

Universidade Federal do Paraná | Programa de Pós-Graduação em Comunicação

de comunicação na contemporaneidade, precisa modelar-se ao novo contexto, mais desafiador (LOPEZ, 2009).

Num panorama de um cenário feito de cenários, a tecnologia e seu franco progresso ampliado no cotidiano da atividade e do trabalho humano é facilmente indicada como a promotora de transformações (URIBE, 2006). O fenômeno tecnológico está ligado a uma atmosfera fundamentalmente sociocultural anteriormente (BURAFAH JUNIOR, 2009). Numa oferta exponencial de ferramentas e plataformas tecnológicas, tem-se, sobretudo, um movimento intimamente humano, social, cultural e profundamente contemporâneo no hoje (LÉVY, 2000). Cada esboço tecnológico, de mudança de paradigmas, de rompimento de eixos convencionais de processamento da informação e da interação humana se caracteriza como um espaço privilegiado de acuidade humana (KISCHINVSKY, 2007). De um lado um plano técnico vislumbrado no cotidiano humano e social, de outro lado perspectivas socioculturais que provocam implicações e transformações substanciais, com imbricações fenomenológicas mútuas (LÉVY, 2000; BURAFAH JUNIOR, 2009).

A evolutiva tecnológica deve ser entendida como participante de uma frente fenomenológica no tempo e no espaço de reorganização dos eixos convencionais, da convergência de sinais, produtos e abordagens (LOPEZ, 2009). O rádio, objeto empírico desta investigação, diante do fluxo oceânico de transformações oriundas da convergência, tem não apenas sua agenda, mas sua própria existência constitutiva modificada (TEIXEIRA; SILVA, 2009; VIGIL, 2003).

No solo da cibercultura e no trâmite técnico, os meios de comunicação como o rádio, têm sua estrutura remodelada sob as sinergias de um espaço ressignificado (LÉVY, 2000; VIGIL, 2003). A sintonia cede à conexão (KINSCHVISKY, 2007). O surgimento das rádios de plataforma online tem nesse foro fenomenológico sua origem compreendida. É factual que os meios tradicionais modificaram suas relações para tornarem-se mais conectados e atrelados ao universo delineado na cibercultura. Mas ao mesmo tempo, novas iniciativas de produção midiática, na base do ciberespaço, têm crescido e representam um nicho investigativo promissor.

Inserir o rádio em uma plataforma web é o tema central deste trabalho que visa desenvolver um projeto experimental para o veículo de mídia acadêmica das Faculdades Integradas Pitágoras - FIPMoc, a Rádio FIP. A Rádio FIP tem suas transmissões estabelecidas no aparato radiofônico da própria universidade, entretanto sem uma cosmologia digital. A proposta contempla maturar uma série de decisões criativas e estruturais para a transposição do veículo na internet.

A comunicação midiática atinge novas fronteiras constantemente alterando a agenda social e a maneira como os expectadores se relacionam. No tocante a era digital, os media como o rádio podem colher um espectro abrangente de contribuições e posicionar-se de modo mais 


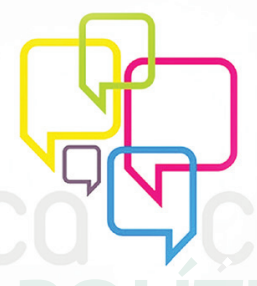

ação mididiátíca

N. 6 | Ano 2013

Universidade Federal do Paraná I Programa de Pós-Graduação em Comunicação

afiado no seio social. Diante dessas exposições, a internet tem gerado frutos cada vez mais expressivos na mediação comunicacional orientando veículos e instituições e, nessa proposição, esse trabalho se justifica por tornar relevantes às discussões e ações entre a comunicação na era digital e os sujeitos sociais.

\section{Métodos}

Estudo qualitativo e descritivo. Percurso desenvolvido através de pesquisa documental orientada a estudar os principais veículos acadêmicos de webrádio para que, num estudo de sua constituição, afluíssem perspectivas criativas e funcionais para a construção do projeto de transposição do veículo tradicional para a internet. A eleição das webrádios foi feita a partir dos veículos que fazem parte da grade do Grupo de Pesquisa Rádio e Mídia Sonora da Sociedade Interdisciplinar de Estudos em Comunicação - Intercom (PORTAL DO RÁDIO, 2013).

As webrádios selecionadas como objeto de análise documental foram a webrádio Frispit da Universidade de Caxias do Sul, Plug Rádio da Universidade Municipal de São Caetano do Sul, Rádio Paralela do Instituto Metodista Izabela Hendrix, RadioFam da Faculdade de Comunicação da PUCRS, Rádio Unesp Virtual da Universidade Estadual Paulista, Rádio Unifor da Universidade de Fortaleza e Rádioweb Unifra do Centro Universitário Franciscano. Na cartela de webrádios, analisaram-se aspectos como estrutura, comunicação visual, constituição e proposta, linguagem, programação, conteúdo e acesso. Tais aspectos foram consultados em vias da elaboração autônoma de um projeto de webrádio acadêmica, como propõe o presente trabalho.

\section{Da análise documental à extração dos dados}

Quanto à constituição e proposta que fundamentam as webrádios, observou-se que os veículos se posicionam como instrumentos de comunicação institucional amplo, para informações institucionais e aproximação do público, em destaque a Rádio Unesp Vistual - UNESP, Rádio Unifor - UNIFOR e Rádioweb Unifra - UNIFRA. Contudo, além da funcionalidade de interface de veículo de comunicação acadêmica, as webrádios também se posicionam como plataformas interativas, de conteúdo elaborado e com ensejo de entretenimento. Nesse ínterim, observamos as webrádios Frispit - UCS, Plug Rádio - USCS, RádioFam - PUCRS e Rádio Paralela - Izabela Hendrix. As decisões criativas recebem fomento da dupla possibilidade de um veículo de comunicação de tal natureza, o diálogo com o público na referência acadêmica e interna e na produção de conteúdo e entretenimento.

Uma preocupação observada foi a interface visual e de acesso das webrádios, uma vez que fundamental o elo inicial com o público ouvinte. Observou-se em todas as webrádios a elucidação de uma estética particular às instituições de que são berçadas, sem exclusão do vínculo. 


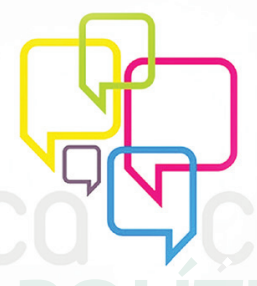

açãô Imidiática

N. 6 | Ano 2013

Universidade Federal do Paraná | Programa de Pós-Graduação em Comunicação

Linguagem editorial e visual próprias são estabelecidas de acordo à criação de cada veículo, sendo o arranjo de layout uma premissa de decisão conceitual do próprio veículo em adequação ao público-alvo ouvinte e sua finalidade. Quando à interface de acesso, são observados plugins de audição próprios bem como a disposição de conteúdo exibido arquivado, para que o público selecione programações antigas através da página de acesso. Comumente, as webrádios analisadas possuem páginas próprias ligadas às instituições matrizes.

O conteúdo abrange caráter de comunicação acadêmica e institucional e prospectos de entretenimento. Observam-se programas noticiosos, informativos, boletins, entrevistas, conteúdo cultural, musical e de entretenimento em geral. Infere-se que embora a diversidade - em função da proposta constitutiva de cada webrádio -, os veículos tramitam numa unidade que tenciona para a unidade acadêmica, sua interação e motivação. Tais observações foram possíveis no conjunto de todas as webrádios tidas em conta na pesquisa proposta.

Tais elementos cunharam um composto reflexivo, no qual as decisões criativas, técnicas e gerais da criação e constituição webrádio para a Rádio FIP foram ancorados. A análise robusteceu a construção do padrão particular do presente projeto, dando vias de embasamento nas iniciativas já adotadas por instituições de expressão e inovação, já que o pensamento criativo aqui traçado teve referência para constituir seu próprio universo.

\section{Da reunião dos dados à constituição de projeto}

O estudo realizado a partir das webrádios selecionadas emergiu a partir da observação e do exame funcional dos veículos, em medidas de compreensão de tais modelos de mídia na internet e em traços de referência que auxiliariam no arranjo criativo da geração de conteúdo e interação para a Rádio FIP. A primeira definição se deu na sedimentação do nome do veículo com Webrádio FIP, indicando os sinais de pertença institucionais acrescidos do elemento indicativo da novidade, a plataforma web.

Os procedimentos do estudo implicaram no desenvolvimento da constituição e programação da Webrádio FIP, favorecendo ainda a criação de sua identidade visual, vinculação eletrônica e amostra de conteúdo - sob aprovação da instituição.

\section{Constituição Webrádio FIP}

Um veículo acadêmico de mídia é, sobretudo, promotor da unidade da comunidade acadêmica, criando elos de interação com o público que se relaciona com a marca institucional. Nesse sentido, as decisões criativas partiram do posicionamento objetivo da Webrádio FIP como veículo difusor dos valores institucionais, promotor de integração e unidade da comunidade acadêmica e gerador de elos de identificação. 

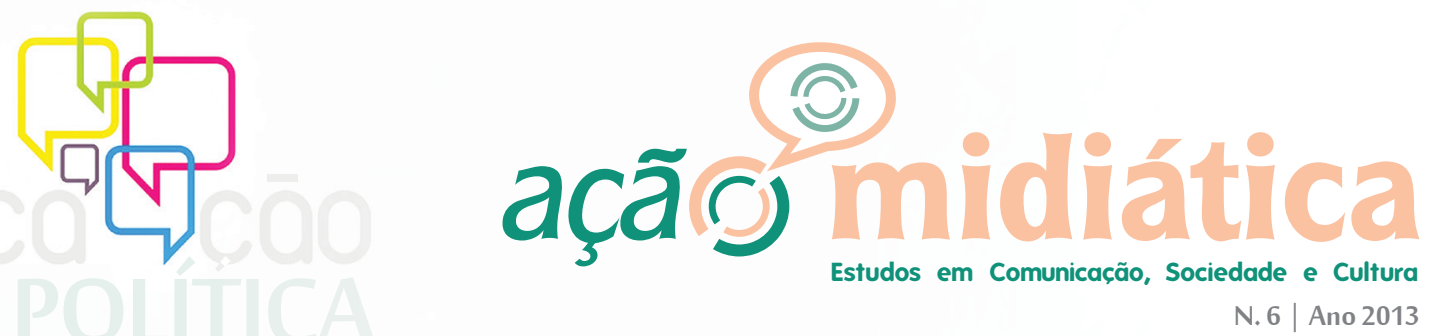

Universidade Federal do Paraná | Programa de Pós-Graduação em Comunicação

\section{Público ouvinte}

Delimitou-se como público, os sujeitos que integram a comunidade acadêmica das FIPMoc por inteiro, entretanto com foco no público entre 18 e 35 anos - faixa etária básica dos acadêmicos - , não se restringindo a docentes e colaboradores da instituição. Há ainda a proposição de que a Webrádio FIP dialogue com o público disposto a partir da idade de 15 anos, público no qual começa a se relacionar com a marca institucional pelo universo do vestibular, das escolhas do futuro e do mercado profissional. São potenciais ingressantes e, na oportunidade do veículo, começarão a relacionar-se com a instituição desde tenro tempo, oportunizando uma estratégia consistente de abordagem e captação.

\section{Estilística}

Uma instituição universitária deve ser entendida inicialmente como uma comunidade singular de pessoas. A Webrádio FIP nesse sentido deve ser um espaço privilegiado de encontro. Quanto ao esquema temático e ao espírito da webrádio, pensou-se posicioná-la como um assertivo de entretenimento. Dessa forma, conecta-se o universo da instituição ao próprio universo pessoal dos sujeitos atendidos, fazendo com que convivam com a marca institucional por mais tempo e em oportunidades recreativas. Com um traçado de entretenimento, o rosto da Webrádio FIP se desenha como cultural, informativo, musical e artístico de maneira personalizada.

\section{Técnica}

A Webrádio FIP será ancorada na tendência contemporânea de conteúdo on demand. Toda a produção de conteúdo e programação será disponibilizada de maneira sistemática ao ouvinte. A webrádio se torna uma experiência de consumo diversificada, onde o ouvinte pode acompanhar os programas e formatos à sua própria escolha e disposição. Uma página própria inserida no portal das FIPMoc conterá não apenas o player e as informações essenciais do veículo, mas num formato hipertexto condensará todo o conteúdo em áudio, texto e vídeo. Um aplicativo permitirá que o público ouvinte selecione através de tags temáticas, programas de conteúdo afim às suas opções de busca, gerando uma playlist personalizada.

\section{Conteúdo}

O teor de programação da Webrádio FIP trafega pelo segmento de entretenimento. Programações diversificadas de entrevistas, talk shows, programas musicais e culturais compõem a grade. Além disso, podcasts temáticos e drops noticiosos da instituição mantenedora recortam a programação. A tabela abaixo expõe o recorte de programação da Webrádio FIP: 


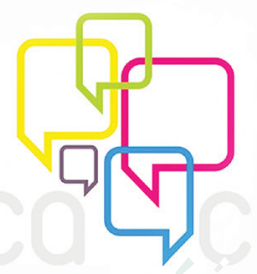

\begin{tabular}{|c|c|}
\hline HORÁRIO & PROGRAMA \\
\hline $6 \mathrm{~h}$ & CAPITÃO CAVERNA: clássicos da música e flashback \\
\hline $7 \mathrm{~h}$ & FACTUAIS: programa noticioso \\
\hline $8 \mathrm{~h}$ & FOLHA DUPLA: revista interativa e temática \\
\hline $10 \mathrm{~h}$ & COUNTDOWN: paradas musicais elegidas pelo público \\
\hline $11 \mathrm{~h}$ & MADBOX: podcast de cultura pop \\
\hline $12 \mathrm{~h}$ & BORA FALAR DE BOLA: esportivo \\
\hline $13 \mathrm{~h}$ & EM VOGA: debates \\
\hline $14 \mathrm{~h}$ & FAZ-ME-RIR: humor e interatividade \\
\hline $15 \mathrm{~h}$ & REPÚBLICA: mercado de trabalho, carreira, cultura \\
\hline $16 \mathrm{~h}$ & FACTUAIS (2 ${ }^{\mathrm{a}}$ EDIÇÃO): programa noticioso \\
\hline $17 \mathrm{~h}$ & TOCA AÍ, UAI: pedidos musicais do público \\
\hline $19 \mathrm{~h}$ & CULTUREBA: agenda cultural \\
\hline $20 \mathrm{~h}$ & MADBOX EXTRA: podcast de cultura pop (estendido) \\
\hline $21 \mathrm{~h}$ & PEQUI DAQUI: talk show de música regional \\
\hline $22 \mathrm{~h}$ & CADEIRA ELÉTRICA: entrevistas \\
\hline $22 \mathrm{~h} 30$ & VEDE!: leitura dinâmica dos fatos do dia \\
\hline $23 \mathrm{~h}$ & THE HEARTBANDS CLUB: musical de bandas consagradas \\
\hline $\mathrm{Oh}$ & FAIXA MUSICAL: Jazz, soul, blues e funk. \\
\hline $1 \mathrm{~h}$ & MADBOX [REPRISE] \\
\hline $2 \mathrm{~h}$ & FAIXA MUSICAL: Pop \\
\hline $3 \mathrm{~h}$ & $\begin{array}{l}\text { REPRISES: } \\
\text { SEGUNDA-FEIRA: República } \\
\text { TERÇA-FEIRA: Cadeira Elétrica } \\
\text { QUARTA-FEIRA: Cultureba } \\
\text { QUINTA-FEIRA: Pequi Daqui } \\
\text { SEXTA-FEIRA: Faz-me-rir }\end{array}$ \\
\hline $4 \mathrm{~h}$ & FAIXA MUSICAL: Nacional \\
\hline $5 \mathrm{~h}$ & FAIXA MUSICAL: Rock \\
\hline SÁBADO & $\begin{array}{l}\text { 6h - Capitão Caverna } \\
7 \mathrm{~h}-0 \mathrm{~h}: \text { Podcasts com o melhor da semana. }\end{array}$ \\
\hline DOMINGO & $\begin{array}{l}\text { 0h }-6 \mathrm{~h}: \text { Faixas musicais } \\
\text { 6h: Capitão Caverna } \\
\text { 7h }-6 \mathrm{~h} \text { (segunda-feira): faixas musicais }\end{array}$ \\
\hline
\end{tabular}

TABELA 1: Programação Webrádio FIP. 


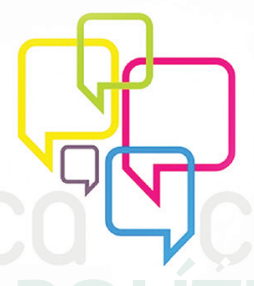

ação midiliática

N. 6 | Ano 2013

\section{Universidade Federal do Paraná I Programa de Pós-Graduação em Comunicação}

\section{Considerações finais}

A sociedade da comunicação vive sob a égide de uma era de convergências, onde a internet é um fator modulador das práticas cotidianas. Os meios de comunicação, como o rádio aqui em questão, ganham novo fulgor e instauram em sua história e agenda uma nova linguagem e abordagem midiática e sociocultural.

A Rádio FIP, veículo de comunicação radiofônica acadêmica das Faculdades Integradas Pitágoras, teve a partir de um estudo documental do fenômeno da cibercultura nas webrádios, um projeto construído para sua transposição à plataforma web. Com forma, conteúdo, estilo e perspectivas constitutivas desenvolvidas, o projeto pode tornar-se um plano expressivo sob o qual a partir do usufruto de um estudo científico, tomando produtos de comunicação como objetos empíricos, puderam gerar no tocante a um cenário tão denso quanto o hodierno.

A resultante final do trabalho culmina num traçado experimental de transposição de um veículo convencional à plataforma web, entretanto, na abstração dos objetivos de pesquisa e no fulgor do percurso metodológico, o presente estudo evidenciou um prisma de possibilidades alicerçadas numa era de convergência comunicacional e onde a cibercultura passa a ser entendida como uma fenomenologia profundamente enraizada no terreno social e próxima dos indivíduos na contemporaneidade. Salienta-se ainda, o potencial integrador e de unidade, os quais, veículos de comunicação como webrádios. Em suma, a substância própria dos mass media como de qualquer produto de comunicação é o próprio substrato sociocultural, numa medida cada vez mais humana no tempo e no espaço.

\section{Referências}

BURAFAH JUNIOR, A. O rádiojornalismo brasileiro diante das ferramentas de interação WEB 2.0. VII Encontro Nacional de Pesquisadores em Jornalismo. São Paulo: USP, novembro de 2009.

KISCHINVSKY, M. O rádio sem onda: convergência e outros desafios de radiodifusão. Rio de Janeiro: E-papers, 2007.

LÉVY, P. Cibercultura. Tradução de Carlos Irineu da Costa. São Paulo: Editora 34, 2000.

LOPEZ, D. C. Radiojornalismo hipermidiático: tendências e perspectivas de rádio all news brasileiro em um contexto de convergência tecnológica. 2009. 299 f. Tese (Doutorado em Comunicação) - Programa de Pós-graduação em Comunicação e Cultura contemporâneas, Universidade Federal da Bahia, Salvador, 2009.

PORTAL DO RÁDIO. Grupo de Pesquisa Rádio e Mídia Sonora da Intercom, 2013. Disponível em: < https://blog.ufba.br/portaldoradio/radios-universitarias/webradios-universitarias/>. Acesso em: 20 nov. 2012. 

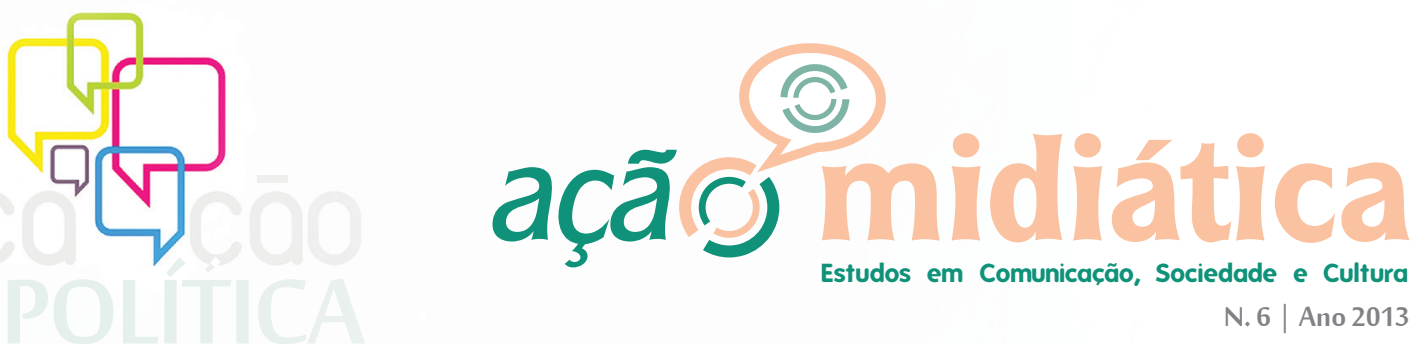

\section{Universidade Federal do Paraná | Programa de Pós-Graduação em Comunicação}

SAMPAIO, R. Educar, ensinar e aprender na era digital: princípios básicos. Acta do X Simpósio Internacional de Informática Educativa. Salamanca, 2008.

TEIXEIRA, M.; SILVA, B. Rádio web: educação, comunicação e cibercultura no universo acadêmico português. Ambientes emergentes. Portugal: Centro de Investigação e Educação Universidade do Minho, 2009.

URIBE, E. V. O rádio digital e o rádio em internet: além das transformações tecnológicas. UniRevista, v. 3, n. 1, jul de 2006.

VIGIL, José Ignácio López. Manual urgente para radialistas apaixonados. Tradução de Maria Luiza Garcia Prada. São Paulo: Paulinas, 2003. 
